\title{
Sexual Satisfaction and Sexual Behaviors During the Covid-19 Pandemic: Results From the International Sexual Health and Reproductive (I-SHARE) Health Survey in Luxembourg
}

\section{Vinicius Jobim Fischer ( $\sim$ viniciusjfischer@gmail.com )}

Research Group Self-Regulation and Health, Institute for Health and Behaviour, Department of Behavioural and Cognitive Sciences, University of Luxembourg

\section{Raquel Gómez-Bravo}

Research Group Self-Regulation and Health, Institute for Health and Behaviour, Department of Behavioural and Cognitive Sciences, University of Luxembourg

\section{Alice Einloft Brunnet}

Laboratoire Clinique Psychanalyse Développement (CLIPSYD - EA4430), University of Paris Nanterre

\section{Kristien Michielsen}

International Centre for Reproductive Health, Dept of Public Health and Primary Care, Faculty of

Medicine and Health Sciences, Ghent University, Ghent

\section{Joseph D. Tucker}

Institute for Global Health and Infectious Diseases, University of North Carolina, Chapel Hill

\section{Linda Campbell}

Centre for Population, Family and Health, University of Antwerp, Antwerp

\section{Claus Vögele}

Research Group Self-Regulation and Health, Institute for Health and Behaviour, Department of Behavioural and Cognitive Sciences, University of Luxembourg

\section{Research Article}

Keywords: sexual satisfaction, sexual behaviors, covid-19, I-SHARE, Luxembourg

Posted Date: January 7th, 2022

DOI: https://doi.org/10.21203/rs.3.rs-1178513/v1

License: (c) (i) This work is licensed under a Creative Commons Attribution 4.0 International License. Read Full License 


\section{Abstract}

Aim: To identify the impact of COVID-19 measures on sexual behaviors and sexual satisfaction in Luxembourg residents.

Methods: We conducted a cross-sectional online survey of adults ( $>18$ years of age) residing in Luxembourg, while COVID-19 restrictions were in place. The survey was available in four languages (French, German, English and Portuguese). Survey questions focused on masturbation, cuddling, condom use, sex frequency, sexting, cybersex, watching porn, and sexual satisfaction.

Results: 557 volunteers completed the survey (35.5\% men, 64.3\% women). Sexual problems increased during the COVID-19 measures while sexual satisfaction decreased compared to prior the COVID-19 measures (assessed retrospectively). Factors associated with increased odds of sexual satisfaction were: having a steady relationship before COVID-19 restrictions, engaging in sexting, reporting good mental health and not altering alcohol intake.

Conclusions: The context of the COVID-19 pandemic and the measures implemented in Luxembourg affected sexual behaviors and sexual satisfaction. Sexual and reproductive health care centers and health professionals in general should take these results into consideration when providing care. Recommendations on the importance of sexual health for general wellbeing and behaviors associated with sexual satisfaction should be offered and possibilities to experience sexuality while reducing contamination risks be discussed.

\section{Introduction}

Since the beginning of the pandemic, the majority of governments around the world began implementing measures to interrupt the spread of SARS-CoV-2. Although varying in scale, these measures impacted people's daily lives. These measures included mandatory use of masks, and restrictions in movement, transit, gatherings, and partial or full confinement (1).

In Luxembourg, after the identification of the first COVID-19 case on March 01, 2020, the government established a pandemic crisis unit and implemented a variety of measures to contain the spread of the virus, including night-time curfew, shutting down services and commerce not considered essential, and mandatory mask use in public spaces. By July 2020, Luxembourg received a score of 45 in the Oxford Stringency Index regarding the stringency of measures introduced to contain the virus (with $0=$ no measure to $100=$ high restrictions) (2). In addition, as a national health policy measure, large-scale testing was offered to the population (3).

Luxembourg is one of the best-performing EU countries in terms of avoiding the worst of the economic impact of the COVID-19 lockdown, in comparison to other EU countries (2). The governmental measures (remote working and schooling, closure of commerce and restaurants, prohibition of social gatherings at home, among others) altered daily living drastically, with implications for individual well-being (4). 
Furthermore, fear of infection or passing the virus on to relatives or friends might elicit feelings of anxiety, depression and other responses (5). For example, an online survey conducted in six European countries in April 2020 found a marked increase in stress, anxiety and depression since the pandemic (4). For cohabiting couples, the lack of possibilities for meeting others, and the reduction in leisure time activities likely increased the amount of time that couples spent together. This could lead to both positive (e.g., strengthening relationships, gratitude, appreciation and tolerance (6) and negative outcomes (e.g. exacerbation of quarrels, couples' bond diminution) (7). It is plausible for such changes to also have affected sexual behaviors and sexual satisfaction.

To date, there is limited evidence on the impact of the COVID-19 pandemic on sexual behaviors. A study from the USA found a diversification of practices but not an increase in frequency of sexual behavior (8). There seems to have been an increase in porn watching and in uploading sexual videos from platforms in different countries (9). Results on sexual frequency are mixed: while Li et al. (10) and Cito et al. (11) found a decrease in sex frequency among young adults in China and in Italy, respectively, Arafat et al. (12) found no change. More conclusive results on sexual frequency would be important, as the lack of sexual activity during the lockdown has been shown to be associated with an increased risk of developing anxiety and depression (13).

Considering the importance of sexual health for general health and wellbeing (14), there is a need to better understand the impact of social restrictions on individuals' wellbeing and their sexuality. To address these and other questions around the impact of COVID-19 related lockdown measures and their effect on sexual behaviors, the I-SHARE consortium was established in early 2020 . This is a collaborative effort including 30 countries with the aim of investigating sexual and reproductive health during the COVID-19 pandemic around the world (15). The present paper presents I-SHARE results from Luxembourg. Luxembourg is one of the most densely populated countries in Europe, with almost half of the population located in the capital city of Luxembourg $(16,17)$.

Multilingualism and multiculturalism are two important facets of Luxembourg's cultural identity, with the five largest foreign communities representing $32.3 \%$ of the total population. Furthermore, the workforce is strongly based on cross-border employees, with around 185,000 employees commuting to Luxembourg daily from France, Germany and Belgium.

The primary aim of the present study was to examine the impact of COVID-19 governmental measures on sexual behaviors, substance use and mental health in Luxembourg. A secondary aim concerned the investigation of factors associated with sexual satisfaction during COVID-19 related measures. As these measures involved several social restrictions, we hypothesized that sexual behaviors would change as a result of these lockdown measures. With respect to in-person partnered sexual activities, we expected to find solo activities and online use for sexual purposes to increase due to the restrictions put in place.

\section{Materials And Methods}




\section{Study population and protocol}

We conducted an online, cross-sectional survey from January 15 to February 12, 2021. The survey was advertised on several University of Luxembourg media platforms (website, Facebook, Instagram and Twitter) and via national agencies, focused on sexual and reproductive health. The questionnaire was available in four languages: French, German, English and Portuguese.

Prior to data collection, ethics approval was obtained from the University of Luxembourg Ethics Review Panel (ERP 20-061-C I-SHARE-Lux), and participants gave consent electronically prior to participation.

Participants met the following inclusion criteria: a) aged 18 years or older, b) residing in Luxembourg and c) fluency in one of the survey languages (French, German, English, Portuguese). All individuals who met the inclusion criteria and had access to the advertisements were invited to participate. No financial compensation or incentive was offered.

The questionnaire was developed collaboratively by the I-SHARE consortium (Michielsen et al., 2021). The same survey instrument was implemented in all countries. In addition, questions could be added for country-specific reasons.

The Luxembourgish versions of the instrument were translated from the English original version into French, German and Portuguese. Participants completed the questionnaire using OpenDataKit (University of Washington, USA) in 10-30 minutes. To complete the survey, participants did not have to respond to all questions, only items associated with skip patterns were mandatory.

Participants were asked to respond to questions concerning sexual behavior and substance use, considering the three months before COVID-19 measures were implemented (pre-COVID-19 measures) and while COVID-19 lockdown measures were in place (during-COVID-19 measures). The sexual behavior questions encompassed: masturbation, sexual intercourse with a steady and/or casual partner, sexting, cybersex and general sexual satisfaction. Sexual satisfaction was addressed with the question "how satisfied were you with your sex life?", and sexual problems with the question: "how often have you or your sexual partner experienced sexual problems?", using 4-point Likert scales $(0=$ not at all satisfied; $3=$ very satisfied, and $0=$ never; $3=$ often, respectively). We also assessed potential changes in substance use frequency between pre- and during-COVID-19 measures concerning alcohol, tobacco, cannabis, cocaine, heroin, and methamphetamine. With respect to mental health, six items assessed emotional states (frustrated, confused, afraid), and cognitive and behavioral changes (obsessive compulsive hand washing, worrying about COVID-19). Response options were: no, neither agree nor disagree, yes. One question enquired about overall mental health perception (good or poor). Questions addressed the present time but also asked if the emotional state or behavior had changed since the COVID-19 measures.

We also included sociodemographic variables (age, sex, education level, number of children) and items on sexuality and relationship status (sexual orientation; general long-term partner variables, i.e. cuddling; 
condom use with casual and/or long-term partners during the specified time period).

\section{Statistical Analyses}

Statistical analyses were conducted using SPSS 26. All participants with valid responses were included. As not all respondents were required to answer all questions, the number of respondents for each question varied.

First, we performed frequency analysis to describe the sample characteristics and the changes on sexual behaviors during the COVID-19 restriction measures in Luxembourg. Second, we performed a paired sample t-test to assess changes in sexual satisfaction and sexual problems before and during COVID-19related restrictions. Lastly, to investigate the factors associated with sexual satisfaction during COVID-19 restrictions, we used a Binary Logistic Regression Model including as predictors demographic characteristics, sexual behaviors and mental health variables. In this step, the variable "sexual satisfaction" was recoded as a binary variable $(0=$ not satisfied and $1=$ satisfied $)$.

\section{Results}

A total of 557 individuals participated in this study, of who 196 (35.2\%) identified their gender as male, $347(62.3 \%)$ as female and $13(2.3 \%)$ as "other", "neither" or "both" genders. Regarding sexual orientation, $429(77 \%)$ reported being heterosexual, $56(10.1 \%)$ bisexual, $30(3.6 \%)$ gay, 13(2.3\%) asexual, $12(2.2 \%)$ pansexual, $6(1.1 \%)$ lesbian, $1(0.2 \%)$ queer, $11(2 \%)$ were questioning or unsure and $6(1.1 \%)$ reported "other" sexual orientation. Regarding couple relationship status, $385(69.1 \%)$ of the participants had a steady partner in the three months before COVID-19. Other demographic characteristics are described in Table 1. 
Table 1

Demographic characteristics of participants.

\begin{tabular}{|c|c|c|c|c|c|}
\hline & & $\mathbf{N}$ & $\%$ & Mean & SD \\
\hline Age $(N=557)$ & & & & 35.9 & 14.4 \\
\hline \multirow[t]{3}{*}{$\operatorname{Sex}(N=557)$} & Man & 198 & 35.5 & & \\
\hline & Woman & 358 & 64.3 & & \\
\hline & Other & 1 & 0.2 & & \\
\hline \multirow[t]{5}{*}{ Gender $(N=556)$} & Man & 196 & 35.2 & & \\
\hline & Woman & 347 & 62.3 & & \\
\hline & Neither & 5 & 0.9 & & \\
\hline & Other & 3 & 0.5 & & \\
\hline & Both & 5 & 0.9 & & \\
\hline \multirow[t]{3}{*}{ Schooling (N=549) } & Primary school & 14 & 2.5 & & \\
\hline & Secondary school & 160 & 28.7 & & \\
\hline & Tertiary school & 375 & 67.3 & & \\
\hline \multirow[t]{9}{*}{ Religion ( $N=555)$} & Buddhist & 1 & 0.2 & & \\
\hline & Hindu & 5 & 0.9 & & \\
\hline & Jewish & 2 & 0.4 & & \\
\hline & Muslim & 9 & 1.6 & & \\
\hline & No religion & 264 & 47.4 & & \\
\hline & Orthodox & 23 & 4.1 & & \\
\hline & Protestant & 16 & 2.9 & & \\
\hline & Roman Catholic & 218 & 39.1 & & \\
\hline & Other & 17 & 3.1 & & \\
\hline \multirow[t]{5}{*}{ Accommodation $(n=555)$} & Flat in flat block & 219 & 39.3 & & \\
\hline & House & 282 & 50.6 & & \\
\hline & Residential/retirement home & 1 & 0.2 & & \\
\hline & Room(s) in shared house & 28 & 5 & & \\
\hline & Student accommodation & 19 & 3.4 & & \\
\hline
\end{tabular}




\begin{tabular}{|lllll|}
\hline & & N & $\%$ & Mean SD \\
\hline Household Income $(n=538)$ & Other & 6 & 1.1 \\
\hline & Prefer not to say & 82 & 15.2 & \\
\hline $0-1250$ & 11 & 2 \\
\hline $1250-2000$ & 23 & 4.3 \\
\hline $2000-4000$ & 31 & 5.8 \\
\hline $6000-8000$ & 84 & 15.6 \\
\hline $8000-12500$ & 13 & 2.4 \\
\hline$>12500$ & 37 & 6.9 \\
\hline
\end{tabular}

The majority of participants ( $n=512,92.2 \%$ ) reported following the COVID-19 measures strictly or very strictly. Regarding COVID-19 testing, 472 (84.7\%) of participants reported a negative COVID-19 test result. Forty-three participants (7.7\%) had tested positive COVID-19 test at least once. Self-reported frequencies of sexual behaviors, sexual problems and sexual satisfaction during the pandemic restrictions are shown in Table 2. The majority of respondents reported no changes in most of their sexual behaviors compared to before the introduction of social distancing measures. For women, behaviors that changed the most were: cuddling with $28 \%$ reporting an increase and $25 \%$ a decrease, sexual activity with a steady partner with $16 \%$ reporting an increase and $39.4 \%$ a decrease, and self-masturbation with almost identical percentages reporting an increase $(20.3 \%)$ or decrease $(20.6 \%)$. For men, smaller changes were observed. Among the sexual behaviors that changed the most were: cuddling (increase reported by $20.9 \%$, and decrease reported by $16.4 \%$ ), sexual activity with a steady partner (increase reported by $11.8 \%$, decrease reported by $28.2 \%$ ), masturbation (increase reported by $29 \%$, decreased reported by $10.2 \%$ ) and watching pornography (increase reported by $29.3 \%$, decrease reported by $7.1 \%$ ). 
Table 2

Sexual behaviors, sexual problems and sexual satisfaction during COVID-19 restriction measures in Luxembourg.

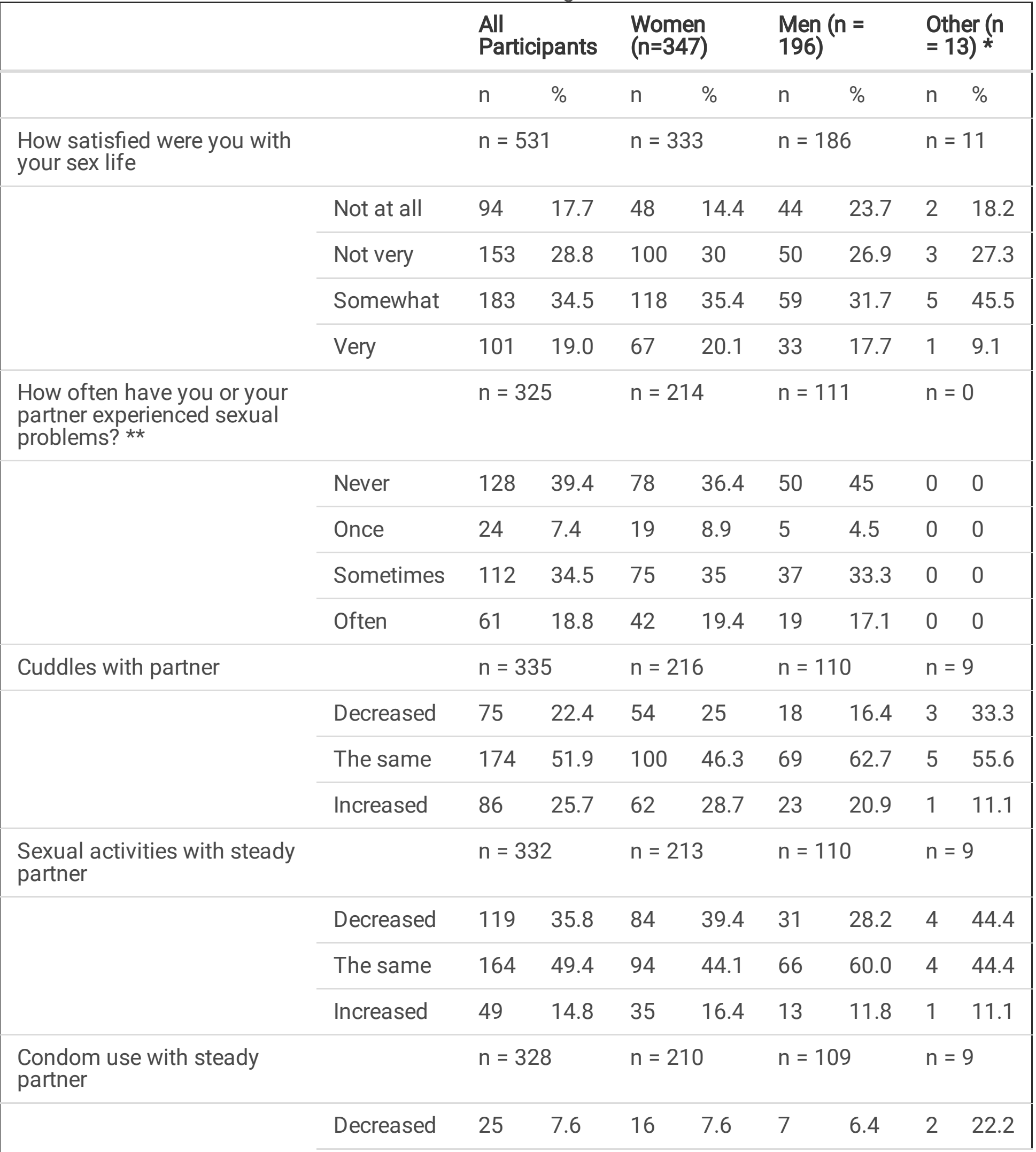

Note. ${ }^{*}$ Other $=$ participants that responded neither or other or both to the question "Which of the following do you identify as?"; ** no participants under the category "Other" responded to this question. 


\begin{tabular}{|c|c|c|c|c|c|c|c|c|c|}
\hline & \multirow[b]{2}{*}{ The same } & \multicolumn{2}{|c|}{$\begin{array}{l}\text { All } \\
\text { Participants }\end{array}$} & \multicolumn{2}{|c|}{$\begin{array}{l}\text { Women } \\
(n=347)\end{array}$} & \multicolumn{2}{|c|}{$\begin{array}{l}\text { Men } \\
196)\end{array}$} & \multicolumn{2}{|c|}{$\begin{array}{l}\text { Other }(n \\
=13) *\end{array}$} \\
\hline & & 299 & 91.2 & 191 & 91 & 101 & 92.7 & 7 & 77.8 \\
\hline & Increased & 4 & 1.2 & 3 & 1.4 & 1 & 0.9 & 0 & 0 \\
\hline \multirow[t]{4}{*}{ Self-masturbation } & & \multicolumn{2}{|c|}{$n=522$} & \multicolumn{2}{|c|}{$n=325$} & \multicolumn{2}{|c|}{$n=186$} & \multicolumn{2}{|c|}{$\mathrm{n}=11$} \\
\hline & Decreased & 89 & 17 & 67 & 20.6 & 19 & 10.2 & 3 & 27.3 \\
\hline & The same & 310 & 59.4 & 192 & 59.1 & 113 & 60.8 & 5 & 45.5 \\
\hline & Increased & 123 & 23.6 & 66 & 20.3 & 54 & 29 & 3 & 27.3 \\
\hline \multirow{4}{*}{$\begin{array}{l}\text { Having sex with casual } \\
\text { partner }\end{array}$} & & \multicolumn{2}{|c|}{$n=509$} & \multicolumn{2}{|c|}{$n=317$} & \multicolumn{2}{|c|}{$n=183$} & \multicolumn{2}{|c|}{$\mathrm{n}=9$} \\
\hline & Decreased & 73 & 14.3 & 34 & 10.7 & 37 & 20.2 & 2 & 22.2 \\
\hline & The same & 401 & 78.8 & 262 & 82.6 & 132 & 72.1 & 7 & 77.8 \\
\hline & Increased & 35 & 6.9 & 21 & 6.6 & 14 & 7.7 & 0 & 0 \\
\hline \multirow{4}{*}{$\begin{array}{l}\text { Condom use with a casual } \\
\text { partner }\end{array}$} & & \multicolumn{2}{|c|}{$n=104$} & \multicolumn{2}{|c|}{$n=46$} & \multicolumn{2}{|c|}{$\mathrm{n}=56$} & \multicolumn{2}{|c|}{$\mathrm{n}=2$} \\
\hline & Decreased & 12 & 11.5 & 8 & 17.4 & 4 & 7.1 & 0 & 0 \\
\hline & The same & 81 & 77.9 & 34 & 73.9 & 45 & 80.4 & 2 & 100 \\
\hline & Increased & 11 & 10.6 & 4 & 8.7 & 7 & 12.5 & 0 & 0 \\
\hline \multirow{4}{*}{$\begin{array}{l}\text { Exchanging naked/semi- } \\
\text { naked pictures, audio or } \\
\text { videos with partner }\end{array}$} & & \multicolumn{2}{|c|}{$n=506$} & \multicolumn{2}{|c|}{$\mathrm{n}=319$} & \multicolumn{2}{|c|}{$\mathrm{n}=178$} & \multicolumn{2}{|c|}{$\mathrm{n}=9$} \\
\hline & Decreased & 39 & 7.7 & 26 & 8.2 & 12 & 6.7 & 1 & 11.1 \\
\hline & The same & 408 & 80.6 & 263 & 82.4 & 138 & 77.5 & 7 & 77.8 \\
\hline & Increased & 59 & 11.7 & 30 & 9.4 & 28 & 15.7 & 1 & 11.1 \\
\hline \multirow{4}{*}{$\begin{array}{l}\text { Watching pornography } \\
\text { videos }\end{array}$} & & \multicolumn{2}{|c|}{$n=515$} & \multicolumn{2}{|c|}{$n=320$} & \multicolumn{2}{|c|}{$\mathrm{n}=184$} & \multicolumn{2}{|c|}{$\mathrm{n}=11$} \\
\hline & Decreased & 56 & 10.9 & 40 & 12.5 & 13 & 7.1 & 3 & 27.3 \\
\hline & The same & 365 & 70.9 & 240 & 75 & 117 & 63.6 & 8 & 72.7 \\
\hline & Increased & 94 & 18.3 & 40 & 12.5 & 54 & 29.3 & 0 & 0 \\
\hline
\end{tabular}

Note. $*$ Other $=$ participants that responded neither or other or both to the question "Which of the following do you identify as?"; ** no participants under the category "Other" responded to this question. 


\begin{tabular}{|c|c|c|c|c|c|c|c|c|c|}
\hline & & \multicolumn{2}{|c|}{$\begin{array}{l}\text { All } \\
\text { Participants }\end{array}$} & \multicolumn{2}{|c|}{$\begin{array}{l}\text { Women } \\
(n=347)\end{array}$} & \multicolumn{2}{|c|}{$\begin{array}{l}\operatorname{Men}(n= \\
196)\end{array}$} & \multicolumn{2}{|c|}{$\begin{array}{l}\text { Other }(n \\
=13) *\end{array}$} \\
\hline \multicolumn{2}{|l|}{$\begin{array}{l}\text { Performed/watched sexual } \\
\text { acts before a webcam }\end{array}$} & \multicolumn{2}{|c|}{$\mathrm{n}=501$} & \multicolumn{2}{|c|}{$\mathrm{n}=315$} & \multicolumn{2}{|c|}{$\mathrm{n}=177$} & \multicolumn{2}{|c|}{$\mathrm{n}=9$} \\
\hline & Decreased & 18 & 3.6 & 13 & 4.1 & 5 & 2.8 & 0 & 0 \\
\hline & The same & 465 & 92.8 & 297 & 94.3 & 160 & 90.4 & 8 & 88.9 \\
\hline & Increased & 18 & 3.6 & 5 & 1.6 & 12 & 6.8 & 1 & 11.1 \\
\hline \multicolumn{10}{|c|}{$\begin{array}{l}\text { Note. * Other = participants that responded neither or other or both to the question "Which of the } \\
\text { following do you identify as?"; ** no participants under the category "Other" responded to this } \\
\text { question. }\end{array}$} \\
\hline
\end{tabular}

Regarding mental health, 42\% $(n=235)$ reported poor overall mental health during COVID-19 restrictions. Participants also reported high rates of frustration because of these restrictions, as well as other negative feelings, which are summarized in Table 3. 
Table 3

Perceptions on COVID-19 and mental health.

\begin{tabular}{|c|c|c|c|}
\hline & & $\mathrm{n}$ & $\%$ \\
\hline \multirow[t]{2}{*}{ How would you rate your overall mental health? } & Good & 320 & 57.5 \\
\hline & Poor & 235 & 42.2 \\
\hline \multirow[t]{3}{*}{ I feel frustrated because of COVID-19 restrictions } & No & 64 & 11.5 \\
\hline & $\begin{array}{l}\text { Nor agree or } \\
\text { disagree }\end{array}$ & 69 & 12.4 \\
\hline & Yes & 422 & 75.8 \\
\hline \multirow[t]{3}{*}{ I am confused about what I can or cannot do due to COVID-19 } & No & 199 & 35.7 \\
\hline & $\begin{array}{l}\text { Nor agree or } \\
\text { disagree }\end{array}$ & 90 & 16.5 \\
\hline & Yes & 266 & 47.8 \\
\hline \multirow[t]{3}{*}{ I am afraid to acquire COVID-19 } & No & 154 & 27.7 \\
\hline & $\begin{array}{l}\text { Nor agree or } \\
\text { disagree }\end{array}$ & 122 & 21.9 \\
\hline & Yes & 280 & 50.5 \\
\hline \multirow{3}{*}{$\begin{array}{l}\text { I experience obsessive or compulsive behaviors with regards to } \\
\text { hand washing }\end{array}$} & No & 334 & 60.0 \\
\hline & $\begin{array}{l}\text { Nor agree or } \\
\text { disagree }\end{array}$ & 128 & 23 \\
\hline & Yes & 94 & 16.9 \\
\hline \multirow[t]{3}{*}{ I cannot stop thinking about the COVID-19 epidemic } & No & 278 & 49.9 \\
\hline & $\begin{array}{l}\text { Nor agree or } \\
\text { disagree }\end{array}$ & 120 & 21.5 \\
\hline & Yes & 158 & 28.4 \\
\hline
\end{tabular}

Sexual problems experienced in the partnership increased significantly during COVID-19 measures, from $M=1.04, S D=1.08$ to $M=1.32, S D=1.17 ; t(324)=-6.52 ; p<001$. Sexual satisfaction decreased significantly during social distancing measures from $M=1.89, S D=0.85$ to $M=1.55 ; S D=0.9 ; t(529)=$ $8.14 ; p<.001$.

The factors associated with increased sexual satisfaction during COVID-19-related measures were assessed with a Binary Logistic Regression Model (Table 4). The results summarized in Table 4 show that participants who were in a steady relationship before COVID-19 restrictions had higher odds of reporting increased sexual satisfaction $(\mathrm{OR}=2.4,95 \% \mathrm{Cl}=1.5-3.7, \mathrm{p}<.00)$. Regarding sexual behaviors, participants who reported an increase in exchanging sex-content messages with a partner had 
higher odds of reporting increased sexual satisfaction $(\mathrm{OR}=2.9,95 \% \mathrm{Cl}=1.0-8.3, \mathrm{p}=.04)$. Those who reported a decrease $(\mathrm{OR}=2.2,95 \% \mathrm{Cl}=1-4.9, \mathrm{p}=.03)$ or no changes $(\mathrm{OR}=2.8,95 \% \mathrm{Cl}=1.5-5.2, \mathrm{p}=$ $.001)$ in self-masturbation, had higher odds of reporting increased sexual satisfaction. 
Table 4

Binary Logistic Regression Model for sexual satisfaction during COVID-19 measures.

\begin{tabular}{|c|c|c|c|c|c|c|}
\hline & & $\mathbf{n}$ & $\boldsymbol{\beta}$ & Wald & $\begin{array}{l}\text { OR } \\
(95 \% \\
\mathrm{Cl})\end{array}$ & $\begin{array}{l}\mathrm{p} \text { - } \\
\text { value }\end{array}$ \\
\hline Age & & 480 & -.02 & 3.24 & $\begin{array}{l}.98 \\
(.96- \\
1.00)\end{array}$ & .07 \\
\hline \multirow[t]{2}{*}{ Sex* } & Men & 170 & -.15 & .45 & $\begin{array}{l}.85 \\
(.55- \\
1.33)\end{array}$ & .49 \\
\hline & Women & 310 & & & & \\
\hline Number of children & & 480 & .09 & .55 & $\begin{array}{l}1.10 \\
(.85- \\
1.42)\end{array}$ & .45 \\
\hline \multirow[t]{3}{*}{ Schooling } & $\begin{array}{l}\text { Primary } \\
\text { school }\end{array}$ & 10 & 1.11 & 2.18 & $\begin{array}{l}3.03 \\
(.69- \\
13.27)\end{array}$ & .14 \\
\hline & $\begin{array}{l}\text { Secondary } \\
\text { school }\end{array}$ & 130 & .46 & 3.78 & $\begin{array}{l}1.58 \\
(.99- \\
2.52)\end{array}$ & .05 \\
\hline & $\begin{array}{l}\text { Tertiary } \\
\text { school }\end{array}$ & 341 & & 5.46 & & .06 \\
\hline \multirow[t]{2}{*}{ Steady partner during COVID-19 } & Yes & 347 & .88 & 14.89 & $\begin{array}{l}2.41 \\
(1.54 \\
- \\
3.78)\end{array}$ & .00 \\
\hline & No & 133 & & & & \\
\hline \multirow[t]{3}{*}{$\begin{array}{l}\text { How often did you have a drink containing } \\
\text { alcohol (during COVID) }\end{array}$} & Decreased & 139 & .20 & .49 & $\begin{array}{l}1.23 \\
(.69- \\
2.20)\end{array}$ & .48 \\
\hline & The same & 235 & .58 & 4.71 & $\begin{array}{l}1.79 \\
(1.05 \\
- \\
3.04)\end{array}$ & .03 \\
\hline & Increased & 106 & & 5.33 & & .07 \\
\hline \multirow[t]{2}{*}{$\begin{array}{l}\text { How would you rate your overall mental } \\
\text { health? }\end{array}$} & Good & 274 & .66 & 9.33 & $\begin{array}{l}1.93 \\
(1.26 \\
- \\
2.96)\end{array}$ & .002 \\
\hline & Poor & 206 & & & & \\
\hline
\end{tabular}




\begin{tabular}{|c|c|c|c|c|c|c|}
\hline & & $\mathrm{n}$ & $\beta$ & Wald & $\begin{array}{l}\text { OR } \\
(95 \% \\
\text { Cl) }\end{array}$ & $\begin{array}{l}\mathrm{p}- \\
\text { value }\end{array}$ \\
\hline \multirow[t]{3}{*}{$\begin{array}{l}\text { I feel frustrated because of COVID-19 } \\
\text { restrictions }\end{array}$} & No & 56 & -.40 & 1.34 & $\begin{array}{l}.66 \\
(.33- \\
1.32)\end{array}$ & .24 \\
\hline & $\begin{array}{l}\text { Nor agree or } \\
\text { disagree }\end{array}$ & 58 & .39 & 1.27 & $\begin{array}{l}1.49 \\
(.74- \\
2.97)\end{array}$ & .26 \\
\hline & Yes & 366 & & 3.16 & & .20 \\
\hline \multirow[t]{3}{*}{$\begin{array}{l}\text { I am confused about what I can or cannot } \\
\text { do due to COVID-19 }\end{array}$} & No & 173 & .32 & 1.62 & $\begin{array}{l}1.38 \\
(.83- \\
2.28)\end{array}$ & .20 \\
\hline & $\begin{array}{l}\text { Nor agree or } \\
\text { disagree }\end{array}$ & 76 & .39 & 1.49 & $\begin{array}{l}1.47 \\
(.79- \\
2.75)\end{array}$ & .20 \\
\hline & Yes & 231 & & 2.30 & & .31 \\
\hline \multirow[t]{3}{*}{ I am afraid to acquire COVID-19 } & No & 134 & -.17 & .42 & $\begin{array}{l}.84 \\
(.49- \\
1.41)\end{array}$ & .51 \\
\hline & $\begin{array}{l}\text { Nor agree or } \\
\text { disagree }\end{array}$ & 105 & .24 & .79 & $\begin{array}{l}1.28 \\
(.74- \\
2.21)\end{array}$ & .37 \\
\hline & Yes & 241 & & 1.90 & & .38 \\
\hline \multirow[t]{3}{*}{$\begin{array}{l}\text { I experience obsessive or compulsive } \\
\text { behaviors with regards to hand washing }\end{array}$} & No & 292 & -.08 & .08 & $\begin{array}{l}.91 \\
(.50- \\
1.66)\end{array}$ & .77 \\
\hline & $\begin{array}{l}\text { Nor agree or } \\
\text { disagree }\end{array}$ & 108 & -.35 & 1.12 & $\begin{array}{l}.69 \\
(.36- \\
1.35)\end{array}$ & .28 \\
\hline & Yes & 80 & & 1.39 & & .49 \\
\hline \multirow[t]{3}{*}{$\begin{array}{l}\text { I cannot stop thinking about the COVID-19 } \\
\text { epidemic }\end{array}$} & No & 241 & .15 & .29 & $\begin{array}{l}1.16 \\
(.66- \\
2.03)\end{array}$ & .58 \\
\hline & $\begin{array}{l}\text { Nor agree or } \\
\text { disagree }\end{array}$ & 98 & .19 & .39 & $\begin{array}{l}1.21 \\
(.66- \\
2.21)\end{array}$ & .52 \\
\hline & Yes & 141 & & .46 & & .79 \\
\hline
\end{tabular}

* Respondent assigned as "other" did not reply to all items in this regression model and therefore was not included in the analysis. 


\begin{tabular}{|c|c|c|c|c|c|c|}
\hline & & $\mathbf{n}$ & $\beta$ & Wald & $\begin{array}{l}\text { OR } \\
\text { (95\% } \\
\text { Cl) }\end{array}$ & $\begin{array}{l}\mathrm{p}- \\
\text { value }\end{array}$ \\
\hline \multirow[t]{3}{*}{ Watching pornography videos } & Decreased & 52 & .17 & .11 & $\begin{array}{l}1.18 \\
(.44- \\
3.16)\end{array}$ & .73 \\
\hline & The same & 339 & .44 & 1.54 & $\begin{array}{l}1.55 \\
(.77- \\
3.12)\end{array}$ & .21 \\
\hline & Increased & 89 & & 1.73 & & .42 \\
\hline \multirow[t]{3}{*}{$\begin{array}{l}\text { Exchanging naked/semi=naked pictures, } \\
\text { audios or videos with partner }\end{array}$} & Increased & 53 & 1.0 & 4.05 & $\begin{array}{l}2.93 \\
(1.02 \\
- \\
8.35)\end{array}$ & .044 \\
\hline & The same & 390 & .74 & 2.93 & $\begin{array}{l}2.09 \\
(.89- \\
4.88)\end{array}$ & .08 \\
\hline & Decreased & 37 & & 4.25 & & .11 \\
\hline \multirow[t]{3}{*}{$\begin{array}{l}\text { Performed/watched sexual acts before a } \\
\text { webcam }\end{array}$} & Increased & 18 & 1.45 & 3.17 & $\begin{array}{l}4.28 \\
(.86- \\
21.25)\end{array}$ & .07 \\
\hline & The same & 444 & .11 & .03 & $\begin{array}{l}1.11 \\
(.33- \\
3.70)\end{array}$ & .85 \\
\hline & Decreased & 18 & & 4.95 & & .08 \\
\hline \multirow[t]{3}{*}{ Self-masturbation } & Decreased & 82 & .81 & 4.24 & $\begin{array}{l}2.26 \\
(1.04 \\
- \\
4.93)\end{array}$ & .039 \\
\hline & The same & 285 & 1.03 & 10.42 & $\begin{array}{l}2.81 \\
(1.50 \\
- \\
5.26)\end{array}$ & .001 \\
\hline & Increased & 113 & & 10.46 & & .005 \\
\hline
\end{tabular}

Good mental health was associated with an increase in sexual satisfaction. Participants who reported "good" general mental health had higher odds of increased sexual satisfaction than those who indicated "poor" mental health $(\mathrm{OR}=1.9,95 \% \mathrm{Cl}=1.2-2.9, \mathrm{p}=.002)$. Concerning alcohol consumption, no changes in self-reported alcohol intake were associated with higher odds for increased sexual satisfaction $(\mathrm{OR}=1.7,95 \% \mathrm{Cl}=1.0-3.0, \mathrm{p}=.03)$. 


\section{Discussion}

The present study examined the impact of measures imposed by the Luxembourgish government to fight the COVID-19 pandemic on self-reported sexual behaviors, substance use and mental health.

We found a decrease in sex frequency during COVID-19 compared to the period before the introduction of the COVID-19 measures. The proportion of respondents reporting a decrease in sexual activities was higher in those with steady partners (35.8\%) compared to those with casual partners $(14.3 \%)$. This decrease was larger than the one found in a study comparing individuals from the English and Spanish populations (7), but smaller than the decrease found in a multi-country study with 30 different countries (18). The reasons for these differences between studies are unclear. Nevertheless, any decrease in sexual activity could have overall health implications, as a decrease in sexual intercourse has been reported to be associated with an overall decline in well-being (11).

A large proportion of the present sample (46.5\%) reported low sexual satisfaction. Similar findings were also found in other I-SHARE countries where, overall, $39.6 \%$ reported low sexual satisfaction (18). This demonstrates the need for health professionals to address sexual and reproductive health issues during the COVID-19 pandemic and the importance of sexual health for general health and wellbeing.

Our data indicate that being in a relationship before the implementation of the COVID-19 measures increased the chances of sexual satisfaction during COVID-19. This is in line with the literature, which found that people in steady relationships during the COVID-19 measures were more sexually active and also more satisfied (18). This corroborates the evidence on the association between sexual satisfaction and more frequent sexual activity $(19,20)$. In addition, this is in accordance with the finding that an increase in self-masturbation was associated with higher odds of increased sexual dissatisfaction. In this context, it is plausible that self-masturbation might have been used as a coping strategy for some. In the present sample, we found a $18 \%$ increase in the frequency of masturbation, which is almost twice the percentage reported by Ibarra et al. (7), but a very similar prevalence of no change in autoerotism when compared to an Italian study (61.2\% in Italy, $59.9 \%$ in Luxembourg) (11).

Findings on the relationship between masturbatory behavior and sexual activity and satisfaction are inconsistent. Masturbation offers the possibility of sexual pleasure independent of a partner's availability and sexual health. For women, masturbation seems to be related to more consistent orgasms compared with partnered sex $(21,22,23)$. Nonetheless, weak or negative associations between solitary and partnered sexual activity or satisfaction have been found $(24,25)$.

With regards to sexual problems, our findings show an increase in sexual problems in those in partnership (either oneself or of the partner) during the pandemic. This result is in line with other studies that have addressed this question. Since the beginning of the pandemic, an increase in sexual problems has been found in a range of populations in different countries, for instance in COVID-19 positive women (26) and in uninfected pregnant women in Turkey (27), in women in the U.S. (28) and in men and women in Egypt (29). 
Our data suggest that people who increased sexting (exchange of sex-content messages such as naked/semi-naked pictures, audios or videos with a partner) had higher odds of reporting sexual satisfaction. Sexting and cybersex might have acted as a tool for different sexual activities in a person or couple's sexual repertoire (30). Our results are in line both with other studies conducted during the COVID19 pandemic that found the use of digital means for sexual communication a way to maintain oneself sexually active (7) and with the literature on sexting and relationship satisfaction that found higher relationship satisfaction in people who engaged in sexting $(31,32,33)$.

With respect to mental health, the ability to adapt to the new pandemic context benefitted levels of sexual satisfaction. This becomes clear when observing that respondents who reported good general mental health presented higher scores of sexual satisfaction. This is in line with the literature, that found a higher risk of developing anxiety and depression among those who were not sexually active during the lockdown (13). Similarly to the adaptation findings regarding mental health, participants who reported no change in alcohol consumption had higher odds of reporting sexual satisfaction. This can possibly be explained by better personal resources to adapt to the pandemic context, and not using alcohol as a coping strategy to deal with the COVID-19 measures. Alcohol consumption as a coping strategy has been identified in previous pandemic outbreaks, e.g. severe acute respiratory syndrome (34). So far, during the COVID-19 pandemic, an increase in alcohol consumption has been reported both in the general population $(35,36)$ and among university students (37).

\section{Strengths and Limitations}

This study has several limitations. First, our study was conducted online which can lead to selection bias (e.g., only people with internet access could take part). Second, our sample was recruited using a convenience approach, predominantly via social media platforms and invitations to key sexual health organizations of the country, which limits the generalizability of the study findings. To overcome such conditions, we employed a broad recruitment strategy (38), using different social media, traditional media, press release, partnering with key governmental and non-governmental institutions as well as invitations to participants of previous COVID19 studies who agreed to be contacted for further studies.

Despite these limitations, this study contributes to the literature on sexual behavior during COVID-19, and the results indicate the importance of continuing research to support policy and help care provision. From a research and policy perspective, longitudinal assessments of the population are needed to properly identify their health needs. On a care provision level, sexual and mental health professionals should be trained and updated to face the population's new demands with reference to sexual behaviors and satisfaction during times of crises such as infectious disease outbreaks.

\section{Conclusions}

The COVID-19 pandemic and the social distancing measures impacted sexual behaviors of the inhabitants of Luxembourg. Sexual and reproductive health care centers and other health professionals 
should take these results into consideration when providing care. Recommendations on the importance of sexual health for general wellbeing and behaviors associated with sexual satisfaction should be offered and possibilities to experience sexuality while reducing contamination risks be discussed.

\section{Declarations}

\section{Ethics approval and consent to participate:}

The present study was conducted in accordance with the Helsinki declaration and its amendments, and in accordance with local legislation. Ethics approval was obtained from the Ethics Review Panel of the University of Luxembourg, Luxembourg (ERP 20-061-C I-SHARE-Lux). All participants gave electronic informed consent to participate and were informed of their possibility to voluntarily terminate their participation at any time.

\section{Consent for publication:}

Consent for publication was obtained from the participants as aggregated and unidentifiable data.

\section{Availability of data and materials:}

The datasets generated and/or analysed during the current study are not publicly available due to the agreement established by the I-SHARE international Consortium parties but are available from the corresponding author on reasonable request.

\section{Competing interests:}

The authors have no competing interests to declare.

\section{Funding:}

the present study received no funding.

\section{Authors' contributions:}

All authors took part on the conceptualization and design of the study. VJF and RGB obtained the register data. AEB created the combined data file and performed the statistical analyses. VJF drafted the manuscript. All authors interpreted the data. All authors reviewed and revised the manuscript critically for its content. All authors read and approved the final version of the manuscript to be published. 


\section{Acknowledgements}

This study was carried out in the context of the I-SHARE study (International Sexual Health And REproductive Health), which examines the impact of the COVID-19 crisis on sexual and reproductive health in diverse low-income, middle-income, and high-income countries. The full list of consortium members and their roles can be found here (https://ishare.web.unc.edu/).

\section{Authors' informations:}

not applicable.

\section{References}

1. Haider N, Osman AY, Gadzekpo A, Akipede GO, Asogun D, Ansumana R, et al. Lockdown measures in response to COVID-19 in nine sub-Saharan African countries. BMJ Glob Heal. 2020 Oct 7;5(10):e003319. Available from: https://gh.bmj.com/lookup/doi/10.1136/bmjgh-2020-003319

2. Luxembourg Research. Luxembourg comes second in dealing with COVID-19. 2021. Available from: https://researchluxembourg.lu/2021/07/12/luxembourg-comes-second-in-dealing-with-covid-19/

3. Luxembourg Research. Large Scale Testing. 2020.

4. Vögele C, Ortmann J, P. C. Lutz A, Schulz A, van Dyck Z, D’Ambrosio C. The impact of social isolation and loneliness on mental health and well-being: the COVID-19 pandemic. In: Self and Society in the Corona Crisis. Esch-sur-Alzette: Melusina Press; 2021. Available from:

https://www.melusinapress.lu/read/the-impact-of-social-isolation-and-loneliness-on-mental-healthand-well-being-the-covid-19-pandemic/section/703c4a5f-94d7-4dfd-8413-37c176972de5

5. Schiavi MC, Spina V, Zullo MA, Colagiovanni V, Luffarelli P, Rago R, et al. Love in the Time of COVID19: Sexual Function and Quality of Life Analysis During the Social Distancing Measures in a Group of Italian Reproductive-Age Women. J Sex Med. 2020;17(8):1407-13. Available from: https://doi.org/10.1016/j.jsxm.2020.06.006

6. Evans S, Mikocka-Walus A, Klas A, Olive L, Sciberras E, Karantzas G, et al. From "It Has Stopped Our Lives" to "Spending More Time Together Has Strengthened Bonds": The Varied Experiences of Australian Families During COVID-19. Front Psychol. 2020 Oct 20;11. Available from: https://www.frontiersin.org/articles/10.3389/fpsyg.2020.588667/full

7. Ibarra FP, Mehrad M, Di Mauro M, Peraza Godoy MF, Cruz EG, Nilforoushzadeh MA, et al. Impact of the COVID-19 pandemic on the sexual behavior of the population. The vision of the east and the west. Int Braz J Urol. 2020;46(Suppl 1):104-12.

8. Lehmiller JJ, Garcia JR, Gesselman AN, Mark KP. Less Sex, but More Sexual Diversity: Changes in Sexual Behavior during the COVID-19 Coronavirus Pandemic. Leis Sci. 2021;43(1-2):295-304. Available from: https://doi.org/10.1080/01490400.2020.1774016 
9. Pornhub Insights. Coronavirus update - June 18. [cited 2021 Sep 2]. Available from: https://www.pornhub.com/insights/coronavirus-update-june-18

10. Li W, Li G, Xin C, Wang Y, Yang S. Challenges in the Practice of Sexual Medicine in the Time of COVID-19 in China. J Sex Med. 2020;17(7):1225-8. Available from:

https://doi.org/10.1016/j.jsxm.2020.04.380

11. Cito G, Micelli E, Cocci A, Polloni G, Russo GI, Coccia ME, et al. The Impact of the COVID-19 Quarantine on Sexual Life in Italy. Urology. 2021;147(January):37-42.

12. Arafat SMY, Alradie-Mohamed A, Kar SK, Sharma P, Kabir R. Does COVID-19 pandemic affect sexual behaviour? A cross-sectional, cross-national online survey. Psychiatry Res. 2020 Jul;289(April):113050. Available from: https://doi.org/10.1016/j.psychres.2020.113050

13. Mollaioli D, Sansone A, Ciocca G, Limoncin E, Colonnello E, Di Lorenzo G, et al. Benefits of Sexual Activity on Psychological, Relational, and Sexual Health During the COVID-19 Breakout. J Sex Med. 2021;18(1):35-49. Available from: https://doi.org/10.1016/j.jsxm.2020.10.008

14. Mitchell KR, Lewis R, O'Sullivan LF, Fortenberry JD. What is sexual wellbeing and why does it matter for public health? Lancet Public Heal. 2021 Aug;6(8):e608-13. Available from: https://linkinghub.elsevier.com/retrieve/pii/S2468266721000992

15. Michielsen K, Larrson EC, Kågesten A, Erausquin JT, Griffın S, Van De Velde S, et al. International Sexual Health and REproductive health (I-SHARE) survey during COVID-19: Study protocol for online national surveys and global comparative analyses. Sex Transm Infect. 2021;97(2):88-92.

16. Luxembourg Government. A small but open society. 2020. Available from: https://luxembourg.public.lu/en/society-and-culture/population/demographics.html

17. Comission E. Eurostat - population density. 2021.

18. Erausquin JT, Tan RKJ, Uhlich M, Francis JM, Kumar N, Campbell L, et al. The International Sexual Health And Reproductive Health Survey (I-SHARE-1): A Multi-Country Analysis of Adults from 30 Countries Prior to and During the Initial COVID-19 Wave. medRxiv. 2021 Jan 1;2021.09.18.21263630. Available from: http://medrxiv.org/content/early/2021/10/19/2021.09.18.21263630.abstract

19. Blair KL, Pukall CF. Can less be more? Comparing duration vs. frequency of sexual encounters in same-sex and mixed-sex relationships. Can J Hum Sex. 2014 Aug;23(2):123-36. Available from: https://utpjournals.press/doi/10.3138/cjhs.2393

20. Higgins JA, Mullinax M, Trussell J, Davidson JK, Moore NB. Sexual Satisfaction and Sexual Health Among University Students in the United States. Am J Public Health. 2011 Sep;101(9):1643-54. Available from: http://ajph.aphapublications.org/doi/10.2105/AJPH.2011.300154

21. Dekker A, Schmidt G. Patterns of Masturbatory Behaviour. J Psychol Human Sex. 2003 Jan 23;14(23):35-48. Available from: http://www.tandfonline.com/doi/abs/10.1300/J056v14n02_04

22. Howard JR, O'Neill S, Travers C. Factors affecting sexuality in older Australian women: sexual interest, sexual arousal, relationships and sexual distress in older Australian women. Climacteric. 2006 Jan 3;9(5):355-67. Available from:

https://www.tandfonline.com/doi/full/10.1080/13697130600961870 
23. Hinchliff S, Tetley J, Lee D, Nazroo J. Older Adults' Experiences of Sexual Difficulties: Qualitative Findings From the English Longitudinal Study on Ageing (ELSA). J Sex Res. 2018 Feb 12;55(2):15263. Available from: https://www.tandfonline.com/doi/full/10.1080/00224499.2016.1269308

24. Brody S, Costa RM. Satisfaction (Sexual, Life, Relationship, and Mental Health) Is Associated Directly with Penile-Vaginal Intercourse, but Inversely with Other Sexual Behavior Frequencies. J Sex Med. 2009 Jul;6(7):1947-54. Available from: https://linkinghub.elsevier.com/retrieve/pii/S1743609515325972

25. Rowland DL, Kolba TN, McNabney SM, Uribe D, Hevesi K. Why and How Women Masturbate, and the Relationship to Orgasmic Response. J Sex Marital Ther. 2020 May 18;46(4):361-76. Available from: https://www.tandfonline.com/doi/full/10.1080/0092623X.2020.1717700

26. Kaya Y, Kaya C, Tahta T, Kartal T, Tokgöz VY. Examination of the effect of COVID-19 on sexual dysfunction in women. Int J Clin Pract. 2021 Mar 27;75(3). Available from: https://onlinelibrary.wiley.com/doi/10.1111/ijcp.13923

27. Karakas LA, Azemi A, Simsek SY, Akilli H, Esin S. Risk factors for sexual dysfunction in pregnant women during the COVID-19 pandemic. Int J Gynecol Obstet. 2021 Feb 23;152(2):226-30. Available from: https://onlinelibrary.wiley.com/doi/10.1002/ijgo.13462

28. Bhambhvani HP, Chen T, Kasman AM, Wilson-King G, Enemchukwu E, Eisenberg ML. Female Sexual Function During the COVID-19 Pandemic in the United States. Sex Med. 2021 Aug;9(4):100355. Available from: https://linkinghub.elsevier.com/retrieve/pii/S2050116121000350

29. Omar SS, Dawood W, Eid N, Eldeeb D, Munir A, Arafat W. Psychological and Sexual Health During the COVID-19 Pandemic in Egypt: Are Women Suffering More? Sex Med. 2021 Feb;9(1):100295.

Available from: https://linkinghub.elsevier.com/retrieve/pii/S2050116120301823

30. Courtice EL, Shaughnessy K. Technology-mediated sexual interaction and relationships: a systematic review of the literature. Sex Relatsh Ther. 2017 Oct 2;32(3-4):269-90. Available from: https://www.tandfonline.com/doi/full/10.1080/14681994.2017.1397948

31. Currin JM, Jayne CN, Hammer TR, Brim T, Hubach RD. Explicitly Pressing Send: Impact of Sexting on Relationship Satisfaction. Am J Fam Ther. 2016 May 26;44(3):143-54. Available from: http://www.tandfonline.com/doi/full/10.1080/01926187.2016.1145086

32. McDaniel BT, Drouin M. Sexting Among Married Couples: Who Is Doing It, and Are They More Satisfied? Cyberpsychology, Behav Soc Netw. 2015 Nov;18(11):628-34. Available from: http://www.liebertpub.com/doi/10.1089/cyber.2015.0334

33. Parker TS, Blackburn KM, Perry MS, Hawks JM. Sexting as an Intervention: Relationship Satisfaction and Motivation Considerations. Am J Fam Ther. 2013 Jan;41(1):1-12. Available from: http://www.tandfonline.com/doi/abs/10.1080/01926187.2011.635134

34. Wu P, Liu X, Fang Y, Fan B, Fuller CJ, Guan Z, et al. Alcohol Abuse/Dependence Symptoms Among Hospital Employees Exposed to a SARS Outbreak: Table 1. Alcohol Alcohol. 2008;43(6):706-12. Available from: https://academic.oup.com/alcalc/article-lookup/doi/10.1093/alcalc/agn073 
35. Vanderbruggen N, Matthys F, Van Laere S, Zeeuws D, Santermans L, Van den Ameele S, et al. SelfReported Alcohol, Tobacco, and Cannabis Use during COVID-19 Lockdown Measures: Results from a Web-Based Survey. Eur Addict Res. 2020;26(6):309-15. Available from: https://www.karger.com/Article/FullText/510822

36. Malta DC, Szwarcwald CL, Barros MB de A, Gomes CS, Machado ÍE, Souza Júnior PRB de, et al. A pandemia da COVID-19 e as mudanças no estilo de vida dos brasileiros adultos: um estudo transversal, 2020. Epidemiol e Serviços Saúde. 2020;29(4). Available from: http://www.scielo.br/scielo.php?script=sci_arttext\&pid=S2237-96222020000400315\&tlng=pt

37. Romero-Blanco C, Rodríguez-Almagro J, Onieva-Zafra MD, Parra-Fernández ML, Prado-Laguna M del C, Hernández-Martínez A. Physical Activity and Sedentary Lifestyle in University Students: Changes during Confinement Due to the COVID-19 Pandemic. Int J Environ Res Public Health. 2020 Sep 9;17(18):6567. Available from: https://www.mdpi.com/1660-4601/17/18/6567

38. Hlatshwako TG, Shah SJ, Kosana P, Adebayo E, Hendriks J, Larsson EC, et al. Online health survey research during COVID-19. Lancet Digit Heal. 2021;3(2):e76-7. Available from: http://dx.doi.org/10.1016/S2589-7500(21)00002-9 